\title{
New antibiotic development: barriers and opportunities
}

\author{
Ercole Concia, Fulvia Mazzaferri, Maddalena Cordioli \\ Complex Operative Unit of Infectious and Tropical Diseases, Policlinico G.B. Rossi, University of Verona, Italy
}

\begin{abstract}
Antibiotic resistance represents a serious threat to public health worldwide, leading to increased healthcare costs, prolonged hospital stays, treatment failures and deaths. To address the emergency of multidrug-resistance, the major international societies of infectious diseases and public health have developed strategies and guidelines to reduce unnecessary antimicrobial use as well as to incite the development of new antibiotics targeting multidrug-resistant pathogens. Even though pharmaceutical companies have been developing new antibiotics since 2010, the global situation is still worrisome. Indeed, the currently available data regarding new antibiotics are limited to microbiological activity and pharmacokinetic profile and their use for the treatment of life-threatening infections (i.e., sepsis) is often off-label. The aim of this article is to present the antibiotic molecules recently commercialized and with which clinicians will deal quite often in next years. We describe ceftolozane/tazobactam, ceftazidime/avibactam, eravacycline, plazomicin, dalbavancin, oritavancin and tedizolid in terms of mechanism of action, antimicrobial spectrum, trials behind the approval and possible indications for the future. In last few years, the US Food and Drug Administration (FDA) and the European Medicines Agency (EMA) approved many new antibiotic molecules but, unfortunately, they lack in biological innovation and in wide clinical indications. These agents show appealing properties for off-label use, as we propose in the article, but caution is still needed considering that high-quality clinical data are limited.
\end{abstract}

\section{Introduction}

According to the Centers for Disease Control and Prevention in the United States (CDC) and to the European Center for Disease Prevention and Control (ECDC), antibiotic resistance is a serious threat to public health worldwide, leading to increased healthcare costs, prolonged hospital stays, treatment failures, and sometimes deaths. In 2013 CDC estimate that approximately 2 million people every year become infected with a multidrug-resistant bacterium, entailing at least 23,000 deaths annually. ${ }^{1}$ Moreover, infections due to multidrug-resistant pathogens are common in patients with well-known risk factors, such as recent

Correspondence: Ercole Concia, Complex Operative Unit of Infectious and Tropical Diseases, Policlinico G.B. Rossi, University of Verona, Piazzale L. Scuro 10, 37134 Verona, Italy. Tel.: +39.0458128243 - Fax: +39.0458128245 .

E-mail: ercole.concia@univr.it

Key words: New antibiotics; sepsis; antimicrobial resistance.

Received for publication: 6 October 2016.

Revision received: 9 November 2016.

Accepted for publication: 9 November 2016.

This work is licensed under a Creative Commons Attribution NonCommercial 4.0 License (CC BY-NC 4.0).

CCopyright E. Concia et al., 2016

Licensee PAGEPress, Italy

Italian Journal of Medicine 2016; 10:255-271

doi:10.4081/itjm.2016.790 antibiotic treatment, presence of prosthetic devices, hospitalization, admission from a nursing home or long-term facilities and the presence of these microorganisms is frequently related to delays in the prescription of an adequate treatment with consequent increases in mortality, length of hospital stay and healthcare costs. ${ }^{2,3}$

In order to address the emergency of multidrug-resistance, the major international societies of infectious diseases and public health have developed strategies and guidelines to reduce unnecessary antimicrobial use as well as to incite the development of new antibiotics targeting multidrug-resistant pathogens. Indeed, while in the past decades the antibiotic advancements have overwhelmed the emergence of new bacterial resistances, in 2010 there were only few antibiotics in the pipeline and they were not enough to face the challenge against multidrug-resistant bacteria.

In recent years, the US White House, the United Kingdom Prime Minister and the European Parliament have stated the necessity of a global effort to reduce the selective pressure for resistant bacteria and to promote and support the pharmaceutical research into new antibiotics. The documents published, which provide for a roadmap for fighting antimicrobial resistance, assembled many different sectors, such as public health, health care, veterinary and agriculture, in order to reduce the antibiotic misuse from the environment and the livestock to the humans. Specifically, the health care's improvement wished by these documents could be translated into the implementation of health care policies and antimicrobial stewardship programs 
that could improve patient outcomes and minimize resistance development by ensuring each patient receives the appropriate antibiotic at the right time, at the correct dose and for the appropriate duration.

While the adoption of antimicrobial stewardship strategies is contributing to the control of the spread of bacterial resistance and although in recent years pharmaceutical companies have developed many new antibiotics, the global situation is still worrisome. Indeed, the indications for these new drugs are limited to the treatment of local infections in adult patients, hence for the treatment of severe, life-threatening infections (i.e., sepsis) the use of these new antibiotics is often off-label. Moreover, for the majority of new antibiotics the currently available data are unfortunately limited to the microbiological activity and pharmacokinetic profile, while information about their effectiveness and safety in vivo is still pending.

The aim of this article is to describe each new antibiotic molecule in terms of mechanism, spectrum, trials behind the approval and its possible indications in the future.

\section{Ceftolozane/tazobactam}

Ceftolozane, previously known as CXA-101 and FR264205, is a new, broad-spectrum cephalosporin that differs from ceftazidime in the presence of a heavier pyrazole ring, an aminothiazole ring and an oxime group that can prevent hydrolysis of the $\alpha$-lactamase active site among AmpC $\alpha$-lactamase over-producing Pseudomonas aeruginosa and confer stability against $\alpha$-lactamases different from $\mathrm{AmpC}$, increasing the inhibitory activity against Enterobacteriaceae species, including Escherichia coli and Klebsiella spp.. ${ }^{4}$ Furthermore, Ceftolozane is not affected by changes in porin permeability and upregulation of efflux pump, antimicrobial resistance mechanisms that are typical of $P$. aeruginosa. ${ }^{5}$ The coformulation with tazobactam, a well-established $\alpha$-lactamase inhibitor, extends the spectrum of activity against the pathogens that harbour class A $\alpha$-lactamases, including most common extended spectrum $\alpha$-lactamases (ESBLs) enzymes such as TEM, SHV, CTX and some class C enzyme such as AmpC, though it is not active against microorganism that produce class A serine carbapenemases and class B metallo- $\alpha$-lactamases. ${ }^{6,7}$

Ceftolozane, as with cephalosporins, exhibits bactericidal activity through the binding of penicillinbinding proteins and, hence, the inhibition of bacterial cell-wall synthesis.

Similar to other cephalosporins, the pharmacodynamics parameter that best predicts bacteriologic efficacy for ceftolozane/tazobactam is time above the MIC (T>MIC as a time-dependent efficacy) for 40 $50 \%$ of the dosing interval. ${ }^{8}$
Pharmacokinetics studies of ceftolozane, when administered alone or in combination with tazobactam as an intravenous infusion, showed a plasma protein binding of approximately $20 \%$ and an extravascular sites distribution. ${ }^{9}$ Moreover, ceftolozane is not a substrate for the cytochrome $\mathrm{p} 450$ system and has no effects on cytochrome p450 enzymes at therapeutic plasma concentrations. Indeed, it has an exclusive renal route of elimination without significant metabolism since its metabolites could be found unchanged in urine $24 \mathrm{~h}$ after the administration. ${ }^{10,11}$ Therefore, subjects with a creatinine clearance equal or lower than $50 \mathrm{~mL} / \mathrm{min}$ or receiving hemodialysis, require a lower daily dose of ceftolozane/tazobactam and, on hemodialysis days, an administration of the drug after completion of dialysis. ${ }^{12}$

In a US multicenter, a surveillance study that included multidrug-resistant $P$. aeruginosa and Enterobacteriaceae spp., ceftolozane/tazobactam remained active in $79 \%$ and $44 \%$ of the isolates, respectively. ${ }^{13}$ This drug, as well as other cephalosporins, has limited activity against anaerobes, both Gram-negative and positive, hence, when a specific anaerobic coverage is required, an additional agent is recommended. Among Gram-positive organisms, ceftolozane/tazobactam is active against Streptococcus species, but has limited activity against Staphylococcus spp. and minimal activity against Enterococcus species. ${ }^{6}$

The clinical efficacy of ceftolozane/tazobactam was evaluated in three phase- 3 trials involving subjects with complicated intra-abdominal infections (ASPECT-cIAI), complicated urinary tract infections (ASPECT-cUTI) and ventilated nosocomial pneumonia (ASPECT-NP).

The ASPECT-cIAI was a double-blind, multicenter, non-inferiority randomized clinical trial that compared ceftolozane/tazobactam [1.5 g intravenously (iv) every $8 \mathrm{~h}$ ] plus metronidazole (500 mg iv every $8 \mathrm{~h}$ ) versus meropenem ( $1 \mathrm{~g}$ iv every $8 \mathrm{~h}$ ) plus placebo for 4-14 days for the treatment of hospitalized cIAI. ${ }^{14}$ In total 806 subjects were eligible for the microbiological intent-to-treat (MITT) analysis and approximately 50\% of the subjects in each group received therapy for up to 7 days. The most common diagnosis was appendiceal perforation and abscess, with the most common pathogen Enterobacteriaceae. Overall, in the ceftolozane/tazobactam plus metronidazole and in meropenem arms, clinical cure was found in $83 \%$ and $87.3 \%$ of patients, respectively, for the MITT population, meeting the primary objective of non-inferiority. In the subgroup analyses of patients with ESBL-producing Enterobacteriaceae, the clinical cure rate was $95.8 \%(23 / 24)$ in the ceftolozane/tazobactam plus metronidazole arm and $88.5 \%(23 / 26)$ in the meropenem one. One-half of the ESBL-producing Enterobacteriaceae isolates at baseline had CTX-M-14 
and CTX-M-15. Of note, although no Klebsiella pneumoniae carbapenemase (KPC)-producing microorganism was isolated in this study, ceftolozane/tazobactam plus metronidazole presented higher activity against antibiotic-resistant strains than meropenem [100\% (13/13) vs $72.7 \%(8 / 11)]$. The incidence of any side effects and the rate of discontinuation were similar in the two groups. The ASPECT-cUTI was a double-blind, noninferiority, randomized clinical trial evaluating ceftolozane/tazobactam ( $1.5 \mathrm{~g}$ iv every $8 \mathrm{~h}$ ) versus levofloxacin ( $750 \mathrm{mg}$ iv once daily) for 7 days for the treatment of complicated lower UTI or pyelonephritis. ${ }^{15}$ The primary efficacy endpoint was defined as complete resolution or marked improvement of clinical symptoms and microbiological eradication at the visit occurred 7 days after last dose in the microbiological modified MITT (mMITT) population, which included the 800 subjects who received medication and had at least one pathogen in the urine. The most common diagnosis was pyelonephritis, with $E$. coli being the most prevalent uropathogen $(78.6 \%)$. In the mMITT population, the clinical cure rate was $76.9 \%$ in the ceftolozane/tazobactam arm and $68.4 \%$ in the levofloxacin one, with this statistically significant difference mainly due to $26.5 \%$ of the pathogens that had baseline resistance to levofloxacin. In a subgroups analysis of subjects with ESBL-producing pathogens, the clinical cure rate was $90.2 \%$ in the ceftolozane/tazobactam arm and $73.7 \%$ in the levofloxacin one. The incidence of adverse effects was similar in both groups and the most common one was headache and gastrointestinal symptoms.

The ASPECT-NP is an ongoing, prospective, double-blind, multicenter, randomized clinical trial which compares ceftolozane/tazobactam ( $3 \mathrm{~g}$ iv every $8 \mathrm{~h}$ ) with meropenem ( $1 \mathrm{~g}$ iv every $8 \mathrm{~h}$ ) for 8 or 14 days (the longer time if the infection was due to $P$. aeruginosa), for the treatment of either ventilator-associated pneumonia or ventilated hospital acquired pneumonia. ${ }^{16}$ This study is still recruiting participants and results will not be available up to October 2018 .

Currently, ceftolozane/tazobactam is approved by US Food and Drug Administration (FDA) and European Medicines Agency (EMA) at the dosage of $1.5 \mathrm{~g}$ iv every $8 \mathrm{~h}$ for 7 days for the treatment of cUTI, in- cluding acute pyelonephritis, and for 4-14 days, in combination with metronidazole $500 \mathrm{mg}$ iv every $8 \mathrm{~h}$, for the treatment of cIAI. The drug is indicated only for infections in adult and it is required caution in patients with renal impairment. ${ }^{17}$ The current renal dosage adjustment recommendations are listed in Table 1.

Ceftolozane/tazobactam demonstrated in vitro activity beyond its currently FDA/EMA-approved indications, including ESBL-producing Enterobacteriaceae and drug-resistant $P$. aeruginosa. However, at this time, clinical data are not sufficient to support its routine clinical use in patients infected with these pathogens.

As for blood stream infections, while few patients with concurrent bacteremia were included in the ASPECT-NP trial, nowadays, there is no knowledge about the use of ceftolozane/tazobactam in this kind of infection. Recently, two papers have been published reporting a successful treatment of sepsis due to multidrug-resistant $P$. aeruginos $a$ with ceftolozane/ tazobactam in an adult $^{18}$ and paediatric ${ }^{19}$ patient, respectively. Further studies and further experiences are needed to clarify the efficacy of ceftolozane/tazobactam in infection other that those approved by FDA/EMA, but already available data on microbiological efficacy and tolerability of this drug make us hope that it would be a precious empiric antibiotic therapy for severe and acute infections in patients with risk factors for multidrug-resistant Gram-negative bacteria.

\section{Ceftazidime/avibactam}

Ceftazidime is a third-generation cephalosporin that was introduced in clinical use in 1985 and it remains, as well other cephalosporins, an important agent in the treatment of bacterial infections because of its broad-spectrum activity, well-characterized pharmacokinetics and pharmacodynamics properties and proven efficacy and safety. Ceftazidime's specific chemical structure, with the presence of an aminothiazole ring and an oxyimino group, strengthens its activity against Pseudomonas spp., while it presents lower activity against Enterobacteriaceae and Grampositive bacteria compared with other third-generation cephalosporins.

Table 1. Renal dose adjustment for ceftolozane/tazobactam.

\begin{tabular}{lc}
\hline Estimated $\mathbf{C r C l}(\mathbf{m L} / \mathbf{m i n}) *$ & Ceftolozane/tazobactam \\
\hline$>50$ & $1.5 \mathrm{~g} / 8 \mathrm{~h}$ \\
\hline 50 to 30 & $750 \mathrm{mg} / 8 \mathrm{~h}$ \\
\hline 29 to 15 & $375 \mathrm{mg} / 8 \mathrm{~h}$ \\
\hline Hemodialysis & LD $750 \mathrm{mg}, \mathrm{MD} 150 \mathrm{mg} / 8 \mathrm{~h}^{\circ}$ \\
\hline$*^{*}$ In the dialysis days, after dialysis; ${ }^{\circ}$ creatinine clearence $(\mathrm{CrCl})$ calculated using the Cockroft-Gault formula. LD, loading dose; $\mathrm{MD}$, mantenance dose.
\end{tabular}

*In the dialysis days, after dialysis; ${ }^{\circ}$ creatinine clearence $(\mathrm{CrCl})$ calculated using the Cockroft-Gault formula. $\mathrm{LD}$, loading dose; MD, mantenance dose. 
Ceftazidime, as with cephalosporins, exhibits bactericidal activity through the binding of penicillinbinding proteins (PBP3 is specifically bound by ceftazidime) and, hence, the inhibition of bacterial cell-wall synthesis.

Avibactam, previously known as NXL104 or AVE1330A, is a new semi-synthetic non- $\alpha$-lactam, $\alpha$ lactamase inhibitor that forms a covalent acetylation of the $\alpha$-lactamase active-site serine residue. This process differs from other $\alpha$-lactamase inhibitor's activity for that fact that while others, that are known as suicide inhibitors, undergo an irreversible reaction producing intermediates that are hydrolysed, the acetylation of avibactam is reversible since it is followed by a process of deacylation that restores its activity. ${ }^{20}$

In vitro avibactam shows activity against class $\mathrm{A}$ $\alpha$-lactamase, including ESBLs and serine kinases (KPC), class $\mathrm{C} \alpha$-lactamase, such as $\mathrm{AmpC}$ and some class D $\alpha$-lactamases, such as OXA-48, while it does not inhibit Ambler's class B metallo $\alpha$-lactamase, such as VIM, NDM, IMP and OXA-type carbapenemases in Acinetobacter spp. owing to the absence of the active-site serine residue. ${ }^{21}$

Addition of avibactam to ceftazidime greatly reduces MICs (4-1024 times) for most Enterobacteriaceae isolates compared with ceftazidime alone and it also showed activity against ceftazidime-resistant $P$. aeruginosa. ${ }^{22,23}$ As showed in the paper written by Sader et al., ceftazidime/avibactam can inhibit a very high percentage and broader range of Enterobacteriaceae that harbour ESBLs, including carbapenemases. Among all Pseudomonas isolates, ceftazidime/avibactam was effective against $99.6 \%$ of them and in a subgroup of isolates with ceftazidime-resistance or meropenem-resistance, the susceptibility rates of ceftazidime-avibactam were reduced to $82.1 \%$ and $87.3 \%$ respectively. ${ }^{24}$ In a further study regarding the activity of this drug against multidrug-resistant and extremely drug-resistant $P$ seudomonas strains, its susceptibility rates were $81 \%$ and $74 \%$ respectively. ${ }^{25}$ As written above, ceftazidime/avibactam has only minimal activity against Acinetobacter spp., Gram-negative bacteria that harbour metallo $\alpha$-lactamases, anaerobic and Gram-positive bacteria. ${ }^{26}$

Similar to other cephalosporins, the pharmacodynamics parameter that best predicts bacteriologic efficacy for ceftazidime/avibactam is time above the MIC ( $>$ MIC as a time-dependent efficacy) for $40-50 \%$ of the dosing interval. Pharmacokinetics studies demonstrated that protein binding of ceftazidime/avibactam is lower than $10 \%$ and that $80-90 \%$ of the drug is excreted unchanged in the urine in the first $6 \mathrm{~h}^{27,28}$

The clinical efficacy and safety of ceftazidime/ avibactam in cIAI and cUTI was assessed by two-phase 2 and 3 clinical trials recently published. ${ }^{29-32}$ In the prospective, double-blind, multicenter, randomized clinical trial conducted for patients with cIAI requiring surgical intervention and antibiotics, ${ }^{33} 303$ subjects were enrolled and then split into two groups receiving either ceftazidime/avibactam ( $2.5 \mathrm{~g}$ iv every $8 \mathrm{~h}$ ) plus metronidazole (500 $\mathrm{mg}$ iv every $8 \mathrm{~h}$ ) or meropenem (1 $\mathrm{g}$ iv every $8 \mathrm{~h}$ ) plus placebo for a minimum of 5 days and a maximum of 14 days. The most common isolates were Gram-negative bacteria, first of all $E$. coli, which were susceptible to both ceftazidime/avibactam and meropenem in $100 \%$ of cases. A successful clinical response was observed in $91.2 \%$ and $93.4 \%$ of ceftazidime/avibactam plus metronidazole and meropenem plus placebo groups, respectively, with an estimated difference in response rate of $-2.2 \%$. $(95 \%$ confidence interval (CI): $-20.4 \%$ to $12.2 \%$ ). Moreover, although low rates of resistant isolates, a good response was found also in among ceftazidime-non-susceptible bacteria $(96.2 \%$ and $94.1 \%$ in the ceftazidime/avibactam plus metronidazole and meropenem plus placebo arm respectively). ${ }^{33}$

In the phase 2, prospective, double-blind, multicenter, randomized trial conducted in patients with cUTI, ceftazidime/avibactam ( $0.625 \mathrm{mg}$ iv every $8 \mathrm{~h}$ ) was compared to imipenem/cilastatin (500 $\mathrm{mg}$ iv every $6 \mathrm{~h}$ ) for a total of 7 to 14 days, with the possibility to switch to oral ciprofloxacin at least after 4 days of iv treatment. ${ }^{29}$ The most isolated pathogen was E. coli and while $67.2 \%$ of isolated bacteria were susceptible to ceftazidime, all of them were susceptible to imipenem. Favourable microbiological response was achieved in $70.4 \%$ of patients treated with ceftazidime/avibactam and $71.4 \%$ of those receiving imipenem/cilastatin [observed difference $-1.1 \%$ (95\% CI: $-27.2 \%$ to $25.0 \%)] .{ }^{29}$ Since in both these phase-2 trials there were too few drug-resistant pathogens, a phase-3 study (REPRISE) has been set up in order to assess the efficacy of ceftazidime/avibactam ( $2.5 \mathrm{~g}$ iv every $8 \mathrm{~h}$ ) compared with the best available therapy (carbapenem in $97 \%$ of cases) in cIAI and cUTI due to ceftazidime-resistant pathogens. The results, recently published, showed no inferiority of ceftazidime/avibactam treated arm in terms of efficacy, safety and tolerability. ${ }^{34}$

The RECLAIM-1 and RECLAIM-2 trials were phase- 3 studies evaluating the treatment with ceftazidime/avibactam ( $2.5 \mathrm{~g}$ iv every $8 \mathrm{~h}$ ) plus metronidazole $(500 \mathrm{mg}$ iv every $8 \mathrm{~h}$ ) compared with meropenem $(1 \mathrm{~g}$ iv every $8 \mathrm{~h})$ in cIAI. The study randomized 1066 patients in the two arms of treatment and, in the end, ceftazidime/avibactam was found to be non-inferior to meropenem in clinical cure rate for the three analyzed populations (modified intention-totreat, clinically evaluable and microbiologic modified intention-to-treat). ${ }^{30}$ It is important to underline that in this trial a subgroup analysis was performed on patients with a moderate renal impairment [creatinine 
clearance $(\mathrm{CrCl})$ between 50 al $30 \mathrm{~mL} / \mathrm{min}]$ at baseline. This analysis revealed that patients receiving ceftazidime/avibactam had lower clinical cure rates than those receiving meropenem ( $48.8 \%$ vs $74.4 \%$ respectively). This outcome could possibly have resulted from underdosing ceftazidime/avibactam in patients with renal impairment at baseline since approximately $60 \%$ of those with $\mathrm{CrCl}<50 \mathrm{~mL} / \mathrm{min}$ at baseline, had an estimated $\mathrm{CrCl}>50 \mathrm{~mL} / \mathrm{min} 48 \mathrm{~h}$ later, requiring an increasing in the dosage of ceftazidime/avibactam. This datum probably suggests the need for therapeutic drug monitoring to adjust ceftazidime/avibactam dosage in patients with renal impairment.

Others phase-3, double-blind, randomized studies have recently been completed but their results are not yet available: the RECAPTURE- 1 and RECAPTURE2 evaluated the effects of ceftazidime/avibactam comparing doripenem for treating cUTI, ${ }^{31,32}$ while another trial assessed the efficacy, safety and tolerability of ceftazidime/avibactam ( $2.5 \mathrm{~g}$ iv every $8 \mathrm{~h}$ ) compared to meropenem ( $1 \mathrm{~g}$ iv every $8 \mathrm{~h}$ ) in the treatment of nosocomial pneumonia, including ventilator-associated pneumonia. ${ }^{35}$ Other clinical trials are currently recruiting patients in order to gain pharmacokinetics data (PK/PD parameters in critically ill patients, ${ }^{36}$ steady state PK in cystic fibrosis, ${ }^{37}$ ceftazidime/avibactam concentration in epithelial lining fluid and plasma, ${ }^{38}$ etc.) and to assess the efficacy and tolerability of the drug in the paediatric population..$^{39,40}$

Currently, the US FDA approved in February 2015 the use of ceftazidime/avibactam for the treatment of cIAI (in combination with metronidazole) and cUTI, in adult patients. In July 2016, EMA extended the FDA indications for the clinical use of ceftazidime/avibactam, including hospital-acquired pneumonia and ventilator-associated pneumonia and infections due to aerobic Gram-negative organisms in adult patients with limited treatment options.

The FDA and EMA indication for the clinical use of ceftazidime/avibactam and the dosage for patient with normal renal function $(\mathrm{CrCl} \geq 51 \mathrm{~mL} / \mathrm{min})$ are listed in Table 2, while Table 3 presents the recommended dosage for patients with renal impairment. ${ }^{41,42}$

Other than clinical trials, there is only little published experience in the use of this drug for the treatment of multidrug-resistant organisms (i.e., carbapenemase-producing bacteria). Gallagher et al. presented an encouraging abstract with a case series of five patients treated with ceftazidime/avibactam on compassionate use with infections due to carbapenemresistant $K$. pneumoniae ${ }^{43}$ and Camargo et al. described a successful treatment of carbapenemase-producing pandrug-resistant $K$. pneumoniae bacteraemia. ${ }^{44}$ Based on these experiences, ceftazidime/avibactam shows en-

Table 2. US Food and Drug Administration/European Medicines Agency approved indications for ceftazidime/avibactam and its dosage for patients with normal renal function (creatinine clearance $\geq 51 \mathrm{~mL} / \mathrm{min}$ ).

\begin{tabular}{lcccccc}
\hline Type of infection & FDA indication & EMA indication & Dosage & Frequency & Infusion time (h) & Duration of treatment \\
\hline cIAI & Yes & Yes & $2.5 \mathrm{~g}$ & Every $8 \mathrm{~h}$ & 2 & $5-14$ days \\
\hline $\begin{array}{l}\text { cUTI (including } \\
\text { pyelonephritis) }\end{array}$ & Yes & Yes & $2.5 \mathrm{~g}$ & Every $8 \mathrm{~h}$ & 2 & $5-10$ days \\
\hline HAP (including VAP) & No & Yes & $2.5 \mathrm{~g}$ & Every $8 \mathrm{~h}$ & 2 & 2 \\
$\begin{array}{l}\text { Infection due to } \\
\begin{array}{l}\text { Gram-negative bacteria } \\
\text { in patients with limited } \\
\text { treatment options }\end{array}\end{array}$ & No & Yes & $2.5 \mathrm{~g}$ & Every $8 \mathrm{~h}$ & $\begin{array}{c}\text { Guided by the severity } \\
\text { of the infections, the pathogen } \\
\text { and the patient's clinical } \\
\text { and bacteriological progress }\end{array}$ \\
\hline
\end{tabular}

FDA, Food and Drug Administration; EMA, European Medicines Agency; cIAI, complicated intra-abdominal infections; cUTI, complicated urinary tract infections; HAP, hospital acquired pneumonia; VAP, ventilator-associated pneumonia.

Table 3. Recommended dosage of ceftazidime/avibactam for patients with renal impairment.

\begin{tabular}{lccc}
\hline Estimated $\mathbf{C r C l}(\mathbf{m L} / \mathbf{m i n}) *$ & Dosage & Frequency & Infusion time (h) \\
\hline 50 to 31 & $1.25 \mathrm{~g}$ & Every $8 \mathrm{~h}$ & 2 \\
\hline 30 to 16 & $0.94 \mathrm{~g}$ & Every $12 \mathrm{~h}$ & 2 \\
\hline 15 to 6 & $0.94 \mathrm{~g}$ & Every $24 \mathrm{~h}$ & 2 \\
\hline$\leq 5^{\circ}$ & $0.94 \mathrm{~g}$ & Every $48 \mathrm{~h}$ & 2 \\
\hline
\end{tabular}

* On hemodialysis days, administer the drug after completion of hemodialysis; ${ }^{\circ}$ creatinine clearence $(\mathrm{CrCl})$ as calculated using the Cockroft-Gault formula. 
couraging data for the treatment of carbapenem-resistant bacteria Gram-negative.

\section{Eravacycline}

Eravacycline, previously known as TP-434, is the first novel synthetic fluorocycline that contains the typical tetracycline structure but that is characterized by the presence of a fluorine atom at $\mathrm{C} 7$ and a pyrolidinoacetamido group at $\mathrm{C} 9 .{ }^{45}$ These structural differences make eravacycline able to overcome tetracycline-specific resistance mechanism such as active efflux pump and ribosomal protection proteins or ribosome hydrolysis. ${ }^{46}$ As well as other tetracyclines, eravacycline inhibits bacterial protein synthesis through binding to the $30 \mathrm{~S}$ ribosomal subunit and its microbiological activity includes Gram-positive and Gram-negative drug-resistant bacteria and anaerobes. Indeed, eravacycline demonstrates a potent activity against staphylococci, both methicillin susceptible and resistant, enterococci, including vancomycin-resistant Enteroccous faecium, and streptococci. ${ }^{47}$ Moreover, eravacycline shows activity against a wide spectrum of Gram-negative multidrug-resistant pathogens such as ESBL-producing and carbapenemase-producing Enterobacteriaceae and Acinetobacter baumannii, against which eravacycline exhibits a fourfold higher activity compared to tigecycline, ${ }^{48}$ but, similarly to tigecycline, eravacycline is not active against $P$. aeruginosa.$^{47}$ Recently, the activity of eravacycline against carbapenem-resistant and tigecycline-resistant Enterobacteriaceae and A. baumannii has been assessed through two in vitro studies: both of them stated that eravacycline MICs correlates closely with those of tigecycline, but mostly were twofold lower. ${ }^{49,50}$

One of the most interesting characteristics of this new drug is the availability of both oral and intravenous formulations, representing an attractive option for early oral switch from intravenous therapy. Intravenous eravacycline exhibits linear, dose-dependent activity and a non-linear, concentration-dependent protein binding profile, as reported in tigecycline, that ranges from 12.5 to $97.3 \%$ (mean $71.4 \%$, standard deviation $17.1 \%) .{ }^{51}$

Eravacycline's bacterial killing profile has been recently assessed through a pharmacokinetic study on a murine thigh infection model with two different methicillin-resistant $S$. aureus (MRSA) and three Enterobacteriaceae isolates, compared to linezolid, vancomycin and tigecycline..$^{52}$ Authors demonstrated that, although eravacycline, as well as other antibiotics with ribosome-mediated mechanism of action, is considered bacteriostatic, its killing effect varies with the species being tested, performing as a bactericidal drug (at least 3-log reduction in bacterial density over a 24$\mathrm{h}$ period) against MRSA within $48 \mathrm{~h}$ whereas van- comycin, tigecycline and linezolid achieved the same result after 72 h. ${ }^{52}$

As described by Leighton et al. in the poster presented at the $21^{\text {st }}$ European Congress of Clinical Microbiology and Infectious Diseases, the oral bioavailability of oral solution of eravacycline ranges from 26 to $32 \%$ with a mean of $28 \% .^{53}$

The phase 1 study published by Connors et al. evaluating the distribution of eravacycline in blood and bronchoalveolar lavage, shows an interesting ability of the drug to reach and concentrate in lungs. Indeed, after a total of 7 doses of drug $(1 \mathrm{mg} / \mathrm{kg}$ iv every $12 \mathrm{~h}$ ) the concentrations of eravacycline in epithelial lining fluid and in alveolar macrophages were 6-fold and 50-fold higher than in plasma, respectively. ${ }^{54}$

Clinical efficacy and safety of eravacycline (1 $\mathrm{mg} / \mathrm{kg}$ iv every $12 \mathrm{~h}$ or $1.5 \mathrm{iv} \mathrm{mg/kg} \mathrm{every} 12 \mathrm{~h}$ ) in cIAI was evaluated in a phase 2 clinical trial with ertapenem ( $1 \mathrm{~g}$ iv every $24 \mathrm{~h})$ as comparator. In all groups the clinical response was above $90 \%$ and, analysing the subgroup of ESBL-producing pathogens, the successful response was $80 \%, 100 \%$ and $100 \%$, respectively. ${ }^{55}$ Since this study demonstrated that the efficacious dose of eravacycline was 1 $\mathrm{mg} / \mathrm{kg}$ iv every $12 \mathrm{~h}$, the following IGNITE- 1 phase 3 , prospective, double-blind, multicenter, randomized trial demonstrated the non-inferiority of this dosage of eravacycline to $1 \mathrm{~g}$ iv every $24 \mathrm{~h}$ compared to ertapenem ( $1 \mathrm{~g}$ iv every $24 \mathrm{~h}$ ) for the treatment of cIAI. ${ }^{56}$ At the same time, the IGNITE-2, another phase 3, prospective, multicenter, double-blind, non-inferiority randomized trial was comparing the clinical efficacy of eravacycline $(1.5 \mathrm{mg} / \mathrm{kg}$ iv every $24 \mathrm{~h}$ followed by $200 \mathrm{mg}$ os every $12 \mathrm{~h}$ ) and levofloxacina (750 $\mathrm{mg}$ iv every $24 \mathrm{~h}$ followed by $750 \mathrm{mg}$ os every $24 \mathrm{~h}$ ) for the treatment of cUTI. ${ }^{57}$ Each patient received a minimum of three days of intravenous treatment and then, if clinically indicated, the therapy could be switched to oral regimen for the remaining dose for a total treatment of 7 days. Even though the enrolment of participant for IGNITE-2 is over and the trial's primary end-point of statistical non-inferiority compared to iv/os levofloxacin was not achieved, more analyses are still ongoing in order to assess the reasons for this result. ${ }^{58}$

The most common adverse events reported in clinical trials were, as well as during tigecycline treatment, mild gastrointestinal symptoms such as nausea and vomiting. ${ }^{56}$

The in vivo efficacy of eravacycline and its possibility to be an attractive option for the treatment not only of cIAI and cUTI but also for other serious infections with a high incidence of drug-resistant Gram-positive and Gram-negative bacteria, was demonstrated by murine model study performed by Grossmann et al. ${ }^{59}$ Indeed, Grossmann and colleagues found that eravacycline was as effective as linezolid and more effective 
than vancomycin in MRSA lung infection models and that, in lung infections due to tetracycline-resistant Streptococcus pneumoniae, eravacycline was able to be even more effective than linezolid. ${ }^{59}$

In conclusion, eravacycline is a promising antibiotic that extends the clinical efficacy and use of tetracyclines against multidrug-resistant Gram-negative and positive bacteria with mild common side effects and the possibility of an early switch from a twicedaily intravenous therapy to an oral one. Even though eravacycline has not yet been approved by both the FDA and the EMA and, FDA asked for the IGNITE-2 results to support a new drug application, it seems to be credible that the use of eravacycline would be precious as an effective carbapenem-sparing regimen for severe infection (not only cIAI and cUTI) due to Gram-negative resistant pathogens.

\section{Plazomicin}

The aminoglycosides, one of the oldest classes of antibiotics, have a broad spectrum of activity and a bactericidal action due to the inhibition of protein synthesis and the subsequent death of the cell. They have often been used in combination with $\alpha$-lactams or quinolones for the treatment of severe infection, mainly due to Gram-negative bacteria. In recent years, their therapeutic use has been limited by concerns of toxicity, such as nephrotoxicity and ototoxicity, and increasing rates of antimicrobial resistance, even though aminoglycosides maintain sufficient level of susceptibility against multidrug-resistant pathogens, compared to other antimicrobials.

Plazomicin, formerly known as ACHN-490, is a next-generation aminoglycoside that has enhanced activity against MRSA and many multidrug-resistant Gram-negative bacteria since it is stable and active against a wide range of the most clinically relevant aminoglycosides-modifying enzymes (AMEs), which are common in ESBL- or carbapenem-producing Enterobacteriaceae.$^{60,61}$ Indeed, plazomicin's chemical structure is distinguished from the one of other classical aminoglycosides by the presence of an unsaturated hydroxyethyl tail and an amino group in the gentamicin ring, which provide no substrate for the AMEs. ${ }^{62}$

Several studies have demonstrated the potential in vitro activity of plazomicin against Gram-positive bacteria, mainly Staphylococcus aureus, and Gram-negative ones, such as Enterobacteriaceae and $P$. aeruginosa ${ }^{49,60}$ In order to fill the gap in the knowledge of plazomicin's efficacy against A. baumannii, Garcia-Salguero and colleagues evaluated its in vitro activity against carbapenem-resistant $A$. baumannii and, in the end, they stated that though they found synergy with several antibiotic combinations, the best one was plazomicin plus carbapenems since this resulted to be active against all strains of multidrug-resistant A. baumannii, indipendently of the carbapenemases possessed. ${ }^{63}$ Presumably, the synergy observed could be the result of the fact that the inhibition of proteins synthesis by the aminoglycosides, which in turn reduces the production of the carbapenemase-enzymes, restores the activity of carbapenems. On the other hand, the evidence that the in vitro concentration of aminoglycosides is 2-fold to 64-fold decreased by the presence of carbapenems may also contribute to reduce aminoglycosides toxicity in humans. ${ }^{63}$ Furthermore, animal studies and two randomized, double-blind, placebo-controlled clinical studies observed only mild to moderate, transient adverse effects and no evidence of nephrotoxicity or ototoxicity. ${ }^{64}$

Plazomicin has a linear and concentration-depending pharmacokinetic profile, with a good penetration in the epithelial lining fluid.

The phase- 2 double-blind, multicenter, randomized, comparator-controlled study in which plazomicin $(10 \mathrm{mg} / \mathrm{kg}$ iv every $24 \mathrm{~h}$ or $15 \mathrm{mg} / \mathrm{kg}$ iv every $24 \mathrm{~h}$ ) was compared to levofloxacin (750 $\mathrm{mg}$ iv every $24 \mathrm{~h}$ ) for the 5-days treatment of cUTI, including acute pyelonephritis, evidenced that the clinical efficacy of the novel aminoglycosides is comparable to the one of levofloxacin ( $88 \%$ vs $81 \%$, respectively). ${ }^{65}$ On the basis of this results, a subsequent phase-3, doubleblind, multicenter, non-inferiority trial is now recruiting subjects in order to compare plazomicin to meropenem for the treatment of cUTI and acute pyelonephritis in terms of clinical and microbiological efficacy and safety. At the same time, another phase3 , open-label, superiority, randomized trial is comparing the efficacy and safety of plazomicin with colistin when combined with a second antibiotic (either meropenem or tigecycline) in the treatment of patients with bloodstream infection, hospital acquired pneumonia, including ventilator-associated pneumonia due to carbapenem-resistant Enterobacteriaceae. ${ }^{66}$

With the results of the phase- 3 trials, expected in late 2017 and 2018 respectively, plazomicin will be able to go through the new drug application process in order to gain the FDA approval and the subsequent possibility to be used in clinical settings.

\section{Dalbavancin}

Dalbavancin, formerly called A40926, is a synthetic lipoglycopeptide that maintains the classical heptapeptide core common to all glycopeptides and, within them, is more similar to teicoplanin, but with differences that enhance its binding affinity and prolong its half-life. Indeed, the chemical structure of dalbavancin is characterized by the addition of a dimethylaminopropylamide group, the lack of the acetylglucosamine group and the substitution of an 
acylaminoglucuronate on ring 4 . These changes also lend a more rapid and powerful activity than with classical glycopeptides among Staphylococcus spp..$^{67,68}$

The mechanism of action of dalbavancin is the inhibition of bacterial cell wall synthesis as a result of the binding of the drug's heptapeptide core to the $\mathrm{C}$ terminal D-alanyl-D-alanyl (D-ala D-ala) of the growing peptidoglycan.

Since dalbavancin has demonstrated no absorption by enteral route, the intravenous administration is the only available with a linear pharmacokinetics profile and a high protein binding property. As regarding the distribution of dalbavancin, Buckwalter and Dowell determined that what describes the best its behaviour is a two-compartment model: within the first $48 \mathrm{~h}$, the concentration of dalbavancin in the central compartment (plasma) rapidly decreases as the drug distributes into the peripheral compartment (tissues), thereby achieving a volume of distribution in tissues at least three times larger than the plasma one ${ }^{69}$ Other studies, detecting the drug in brown fat, kidney, liver, skeletal muscle and skin tissue, stated that dalbavancin appears to distribute relatively equally throughout many tissue..$^{70,71}$

Furthermore, dalbavancin showed no impact on human cytochrome (CY) P450 system and, similarly to other glycopeptides, has a primarily renal excretion. Currently, limited information is available for the use of this antibiotic in patients with renal impairment, but since studies demonstrated that the mean plasma concentration of dalbavancin increases by $11 \%, 35 \%$ and $47 \%$ in patients with mild, moderate and severe renal impairment, respectively, preliminary FDA-approved guidance suggests the dose adjustment listed in Table 4.

The antibacterial activity of dalbavancin is primarily against Gram-positive bacteria. Even though the use of dalbavancin is approved by FDA for the treatment of infection due to $S$. aureus, both methicillinsusceptible and resistant, Streptococcus pyogenes, Streptococcus agalactiae and the Streptococcus anginosus, many in vitro studies revealed that this antibiotic is active against other anaerobes, such as Enterococcus and Lysteria isolates, and anaerobes
Gram-positive bacteria, such as Corynebacterium and Clostridium species. ${ }^{72,73}$ Moreover, the in vitro study performed by Johnson and colleagues for the evaluation of interaction between dalbavancin and others antibiotics used in clinical settings, showed partial synergy, indifference and additive activity in $34.4 \%$, $33.3 \%$ and $22.2 \%$ of simulations, respectively. Moreover, dalbavancin showed a great synergy with oxacillin when used against MRSA. ${ }^{74}$

The clinical efficacy and safety of dalbavancin for the treatment of skin and skin structures infections was assessed in four phase-3 clinical trials. The first one was a double-blind, multicenter, non-inferiority, randomized trial, performed by Jauregui et al. in 2003, comparing dalbavancin (1000 mg day 1 plus $500 \mathrm{mg}$ day 8 ) to linezolid (600 mg every $12 \mathrm{~h}$ ) for 14 days and dalbavancin achieved the statistical non-inferiority endpoint [clinical success $88.9 \%$ vs $91.2 \%$ in the dalbavancin and linezolid group, respectively $(95 \%$ CI lower limit: $-7.3 \%)] .{ }^{75}$ As regard the tolerability, both regimens seem to be safe: participant reported $56 \%$ and $61 \%$ of side effects in the dalbavancin and the linezolid group respectively, but they were mild in the majority of cases since less than $4 \%$ of patients in either treatment regimen discontinued the therapy. ${ }^{75}$

Later, the registrational phase-3, double-blind, multicenter, non-inferiority, randomized trials DISCOVER-1 and DISCOVER-2, compared dalbavancin (1000 mg day 1 plus $500 \mathrm{mg}$ day 8) to vancomycin $(15 \mathrm{mg} / \mathrm{kg}$ every $21 \mathrm{~h})$ for 14 days. Physicians, blinded to the regimen, could switch to oral regimen if clinically possible: oral linezolid $600 \mathrm{mg}$ every $12 \mathrm{~h}$ in the vancomycin arm and oral placebo every $12 \mathrm{~h}$ in the dalbavancin one. The primary endpoint in the intentto-treat population of non-inferiority at the 48-72-h evaluation, was met by dalbavancin. Indeed, the early clinical response was achieved by $79.7 \%$ and $79.8 \%$ of subjects in the dalbavancin and vancomycin group, respectively ( $95 \% \mathrm{CI}:-4.5$ to $4.2 \%$ ) and, by the end of the treatment, almost all subjects were successfully treated $(90.7 \%$ in the dalbavancin arm and $92.1 \%$ in the vancomycin/linezolid one). ${ }^{76}$

Table 4. Dose recommendation of dalbavancin in patients with renal impairment.

\begin{tabular}{|c|c|c|c|}
\hline Regimen & Estimated CrCl (mL/min)* & Dosage (mg) & Infusion time (h) \\
\hline \multirow[t]{3}{*}{ 2-doses } & $>30$ & $1000 \mathrm{mg}$ day 1 plus $500 \mathrm{mg}$ day 8 & 0.5 \\
\hline & $\leq 30$ & $750 \mathrm{mg}$ day 1 plus $375 \mathrm{mg}$ day 8 & 0.5 \\
\hline & Hemodialysis & No adjustment & 0.5 \\
\hline \multirow[t]{3}{*}{ 1-dose } & $>30$ & $1500 \mathrm{mg}$ day 1 & 0.5 \\
\hline & $\leq 30$ & $1125 \mathrm{mg}$ day 1 & 0.5 \\
\hline & Hemodialysis & No adjustment & 0.5 \\
\hline
\end{tabular}

${ }^{*}$ Creatinine clearence $(\mathrm{CrCl})$ as calculated using the Cockroft-Gault formula. 
As found by Jauregui et al., dalbavancin treatment was well tolerated since only $32.8 \%$ of subjects referred adverse effects and, among them, only $2.1 \%$ were serious enough to discontinue the treatment (in vancomycin/linezolid group were $37.9 \%$ and $2.0 \%$, respectively). ${ }^{76}$

On the basis of these promising findings, in 2016 Dunne et al. published the results of another phase-3, double-blind, multicenter, non-inferiority, randomized trial which compared the standard two-doses regimen of dalbavancin ( $100 \mathrm{mg}$ day 1 plus $500 \mathrm{mg}$ day 8 ) to a novel one-dose dalbavancin shot (1500 mg day 1) for the treatment of acute bacterial skin and skin structure infections (ABSSSIs). Since the non-inferiority primary endpoint was achieved (early clinical response in $81.4 \%$ vs $84.2 \%$ in single dose and twodoses regimen, respectively) and severe adverse events were similar between the groups $(1.7 \%$ vs $1.4 \%$ in single dose and two-doses regimen, respectively), the FDA and the EMA recently added to the already approved two-doses regimen of dalbavancin, also the single dose for the treatment of ABSSSIs. ${ }^{77,78}$

The use of dalbavancin in clinical real-life represents many new benefits since, being maximum a 1week-shot treatment, it could reduce the hospital admission and hospital staying and, avoiding the need of a central line, its use could increase patient's compliance and reduce the risk of central venous catheter complication, even in terms of infections.

Currently, dalbavancin is approved only for the treatment of cutaneous infection in adults and off-label use (severe infection due to Gram-positive bacteria such as sepsis, catheter-related infections and osteomyelitis) should be carefully evaluated case by case until more clinical data will be available. Moreover, nowadays many trials are ongoing in order to assess the pharmacokinetics profile, the clinical efficacy and safety for the use of dalbavancin in the pediatric population. ${ }^{79-81}$

\section{Oritavancin}

Oritavancin is a new-generation lipoglycopeptide, structurally similar to vancomycin, but with important modifications from both structural and stereochemical point of view. Indeed, oritavancine's chemical structure is characterized by the presence of both two 4epi-vancosamine monosaccharides, one replacing vancosamine and the other linked to ring-6 via an amino acid residue and a highly hydrophobic $\mathrm{N}$-alkylp-chlorophenylbenzyl substituent linked to the disaccharide sugar. Moreover, the capability of oritavancin to dimerize enhances its ability both to bind and to anchor more effectively the peptidoglycan cell wall of Gram-positive organisms, increasing drug's potency.

Oritavancin exhibits its rapid bactericidal activity through two different mechanism of actions. Indeed, as vancomycin, oritavancin is able to block the transglycosylation and transpeptidation activities, which are essential in peptidoglycan synthesis, but then, differently from vancomycin, oritavancin is able to create a second bind to the pentaglycyl (Asp/Asn) bridging segment within peptidoglycan, which enhances oritavancin's capacity of maintaining binding affinity for the modified peptidoglycan peptide termini of vancomycin-resistant pathogens.

Furthermore, the presence of the hydrophobic group in oritavancin's chemical structure, allows for interaction and disruption of the cell membrane, resulting in depolarization, permeabilization, and concentration-dependent, rapid cell death. This mechanism of action, that oritavancin shares with telavancin but not vancomycin, makes it similar to daptomycin with the difference that oritavancin, with its multiple mechanism of actions, has activity even against daptomycin-nonsusceptible Gram-positive organisms. ${ }^{82}$ Moreover, this membrane-targeted mechanism of action seems to be independent of cellar growth and division as described by Belley and colleagues who analyzed the in vitro activity of oritavancin against MRSA isolates in a non-dividing state. ${ }^{83}$

Hence, from a microbiological point of view, oritavancin demonstrates activity against Gram-positive pathogens, such as staphylococci, including MRSA, hVISA and VISA, streptococci and enterococci, including VRE, Micrococcus spp., Listeria monocytogenes and Corynebacterium spp... ${ }^{84,85}$ In particular, against MRSA, oritavancin has been shown to be 16to 32-fold more potent than vancomycin and linezolid and 8-fold more potent than daptomycin when tested against isolates in both the USA and Europe. ${ }^{86}$

In pharmacokinetics studies, oritavancin displays consistent linear kinetic profile, the bound to serum proteins is around $85-90 \%$ and the distribution into tissues is massive, with a volume of distribution of $87.6 \mathrm{~L}$. After a single dose infusion and the initial distribution phase, less than $10 \%$ of the drug's peak concentration remains in serum $24 \mathrm{~h}$ after, since it is accumulated into tissues and slowly released, presenting a terminal halflife of more than 10 days. Oritavancin is predominantly cleared via the reticuloendothelial system and then slowly excreted unchanged in urine and feces $(<5 \%$ and $1 \%$ of drug is eliminated after 14 days, respectively). ${ }^{87,88}$ Hence, oritavancin does not require dose adjustment in patients with both hepatic and renal dysfunction, even those in hemodialytic treatment, since the drug is not removed by dialysis. ${ }^{89}$

In time-kill experiments, oritavancin exhibits concentration-dependent bactericidal activity by $24 \mathrm{~h}$ against all most common Gram-positive bacteria. ${ }^{90} \mathrm{In}$ triguingly, oritavancin's multiple mechanisms of action confer activity against vancomycin-susceptible and -resistant organisms, as well as rapid, concentra- 
tion-dependent killing versus actively growing, stationary phase, and biofilm-producing Gram-positive organisms.

Oritavancin was evaluated in two phase-3, doubleblind, multicenter, non-inferiority trials, SOLO-1 and SOLO-2, which compared a single-dose of oritavancin (1200 $\mathrm{mg}$ iv followed by place iv every $12 \mathrm{~h}$ for the remaining days of treatment) to vancomycin (15 $\mathrm{mg} / \mathrm{kg}$ iv every $12 \mathrm{~h}$ for 7-10 days) for the treatment of acute bacterial skin and skin structure infections. ${ }^{91,92}$ These trials enrolled a total of 1959 subjects and, notably, rates of MRSA identification were considerable between arms and in both trials $(21.9 \%$ and $20.9 \%$ in SOLO- 1 and $19.9 \%$ and $20.1 \%$ in SOLO-2). In both studies, oritavancin met the non-inferiority primary clinical response endpoint $(82.3 \%$ vs $78.9 \%$ in oritavancin $v s$ vancomycin arms, respectively, in SOLO-1 and $80.1 \%$ vs $82.9 \%$ in oritavancin vs vancomycin groups, respectively, in SOLO-2). Oritavancin met also non-inferiority in two other endpoints in both trials: the additional predefined efficacy one of investigator-assessed clinical cure and the proportion of patients attaining a lesion reduction above $20 \%$. As regard to the safety profile of oritavancin, the two registration trials showed similar rates of adverse effects in both arms and the most common $(\geq 3 \%)$ were nausea, headache and vomiting. Serious side effects were reported only in $5.8 \%$ and $5.9 \%$ of patients on oritavancin and vancomycin, respectively. Compared to vancomycin, oritavancin group had more events of infusion site phlebitis, elevation of ALT levels and limb and subcutaneous abscess.

Intriguingly, oritavancin differentiates to vancomycin in drug-drug and drug-lab interactions. Indeed, in a study that enrolled healthy adult volunteers, oritavancin acted as a non-specific, weak inhibitor of some CYP450 enzymes $(2 \mathrm{C} 9,2 \mathrm{C} 19)$ and as an inducer of others (3A4, 2D6). Hence, when oritavancin was co-administered with warfarin and omeprazole, CYP2C9 and CYP2C19 substrates, it was registered a $31 \%$ and $15 \%$ increase in the warfarin and omeprazole plasma concentration, respectively. ${ }^{89,93}$ In addition, the interaction between oritavancin and CYP3A4, CYP2D6 enzymes resulted in a decreased exposition to midazolam $(-18 \%)$.

Furthermore, oritavancin, due to its ability to bind to the phospholipid reagent, is able to interfere with several laboratory values, such as the prolonged activated partial thromboplastin time and the prolonged prothrombin time/international normalized ratio. These drug-laboratory interactions make the use of these tests unreliable. Nowadays, many trials are ongoing with the aim to better evaluate oritavancin-laboratory interactions and to help clinicians dealing with patients who are on chronic warfarin anticoagulation therapy while they are on oritavancin treatment. ${ }^{94,95}$
In August 2014 and in March 2015, the US FDA and the EMA approved, respectively, the use of oritavancin for the treatment of the acute bacterial skin and skin structure infections in adult. The recommended dosage for healthy adult is a single-dose treatment of $1200 \mathrm{mg}$ iv of drug and no adjustments are required for both hepatic and renal impairment. ${ }^{96,97}$

Despite the FDA and EMA approved labelled indications are narrow, the pharmacokinetic and pharmacodynamic characteristics of oritavancin let clinicians think to this antibacterial agent as an innovative treatment for infections other than the cutaneous ones. Indeed, its broad spectrum and the activity against vancomycin-resistant enterococci, its tolerability profile with an only minimal nephrotoxicity and its easy handling are characteristics that lead clinicians to use this molecule in managing difficult-to-treat Gram-positive infections, such as bacteraemia, endocarditis and osteomyelitis, or infections in fragile subjects, such as those with renal impairment or with low compliance to any kind of multiple-doses antibiotic regimen.

\section{Tedizolid}

Tedizolid, formerly known as TR700, is a secondgeneration oxazolidinone with an expanded-spectrum of activity and a reassuring safety profile. It is commercialized as an inactive prodrug tedizolid phosphate that is rapidly converted in humans to the active compound tedizolid by hydrolysis of the phosphate group. As well linezolid, tedizolid inhibit protein synthesis by binding to the $50 \mathrm{~S}$ ribosomal subunit, hampering aminoacyl t-RNA binding to the A-site of the peptidyl transferase center (PTC)..$^{98,99}$ The PTC binding-site for tedizolid is the same as for linezolid, even though the D-ring of tedizolid may engage additional sites on the ribosome and it is likely responsible for the greater potency than linezolid. ${ }^{100}$

Tedizolid broad spectrum of activity was assessed in many studies, showing that it has great activity against staphylococci, streptococci and enterococci, including multidrug-resistant pathogens, such as vancomycin-resistant, daptomycin-nonsusceptible and linezolid-resistant strains. ${ }^{101-103}$ Compared to linezolid, tedizolid has been shown to have 4- to 16 -fold greater in vitro activity against methicillin-susceptible and -resistant S. aureus, streptococci and enterococci and to maintain its efficacy against linezolid-resistant $S$. aureus with $c f r$ multidrug-resistance gene mutation. ${ }^{101}$ To date, the plasmid-borne ribosomal methyltrasferase gene, $c f r$, has been identified in many Gram-positive and Gramnegative pathogens around the world and will likely continue its spreading because of a low cost of fitness. ${ }^{104}$ Acquisition of the $c f r$ gene leads to a broad cross-resistance to linezolid, as well as to phenicols, lincosamides, streptogramins and macrolides. ${ }^{98,105}$ As re- 
gard to linezolid, $c f r$-mediated methylation of $23 \mathrm{~S}$ rRNA impedes the binding of the antibiotic molecule to its target for steric reasons. Hence, the substitution of the acetamide group with a smaller hydroxymethyl one in tedizolid, allows the binding of the drug and thus activity is retained even against $c f r$-positive isolates. ${ }^{101}$ Furthermore, tedizolid differentiates from linezolid also for the fact that it does not select the retention of the cfr-gene, suggesting a therapeutic advantage for tedizolid when $c f r$-positive strains may be present. ${ }^{106}$

In despite of the activity of tedizolid against susceptible Gram-positive bacteria and linezolid-resistant methicillin-resistant $S$. aureus is maintained, ${ }^{107}$ recently Klupp and colleagues evaluated the in vitro activity of this new oxazolidinon against vancomycinand linezolid-resistant $E$. faecium isolates with $23 \mathrm{~S}$ rDNA mutations. Unfortunately, preliminary results evidenced that, although tedizolid MICs were lower than linezolid ones, they remained above the FDA-approved tedizolid breakpoint $(2-32 \mathrm{mcg} / \mathrm{mL})$, limiting the value of an empiric treatment with tedizolid when a linezolid-resistant vancomycin-resistant $E$. faecium is suspected. ${ }^{108}$

Currently, the antimicrobial susceptibility MIC breakpoints that are available and FDA-approved for tedizolid are against $S$. aureus and the MIC values are $\leq 0.5,1$ and $2 \mathrm{mcg} / \mathrm{mL}$ for susceptible, intermediate and resistant strains, respectively. Moreover, nowadays, no commercially available susceptibility testing method is usable for clinical microbiology laboratories, although a lyophilized broth microdilution system (Sensititre ${ }^{\mathbb{B}}$; Thermo Fisher Scientific, Oakwood Village, OH, USA) seems to be at hand in next future. ${ }^{109}$

As regard the pharmacokinetic profile, tedizolid is a concentration-dependent antimicrobial agent with an equivalent oral/intravenous bioavailability (91\%), a high protein bond (70 to $90 \%$ ) and a large volume of distribution $(67-80 \mathrm{~L})$. It is primarily metabolized in the liver and it is excreted mostly in feces $(82 \%)$. Thanks to the high oral bioavailability, tedizolid is available at the same dosage of $200 \mathrm{mg}$ once daily for both routes of administration and no dose adjustment is required in patients with hepatic or renal impairment. ${ }^{110}$ Furthermore, recently, a pharmacokinetic study of tedizolid in normal weight and obese adults has been published: although the study enrolled only 9 healthy adults in each group, it demonstrated that tedizolid does not require dose adjustment either in morbidity obese adults. ${ }^{111}$

Time-to-kill studies have shown that tedizolid, as other oxazolidinones, has a bacteriostatic activity against both staphylococci, streptococci and enterococci.

The clinical efficacy and tolerability of tedizolid has been assessed in two phase-3, double-blind, multicenter, non-inferiority, randomized trials (ESTABLISH-1 and ESTABLISH-2) which compared a 6-day regimen of tedizolid (200 $\mathrm{mg}$ once daily) to a 10-day therapy with linezolid (600 mg every $12 \mathrm{~h}$ ) for the treatment of adult acute bacterial skin and skin structure infections. ${ }^{112,113}$ The only difference between the two registrational trials was that while the first study compared oral tedizolid to oral linezolid, in the second one subjects received at least two doses of intravenous therapy before allowing a switch to oral regimen of tedizolid or linezolid. In both trials methicillin-resistant $S$. aureus was isolated in considerable and similar rates in the tedizolid and linezolid groups $(42.1 \%$ and $43.1 \%$ in tedizolid and linezolid arms, respectively in ESTABLISH- 1 trial and $27 \%$ and $28 \%$ respectively in the ESTABLISH-2 trial). In both trials, tedizolid met the non-inferiority primary endpoint of clinical early response within 48-72 h. ${ }^{112,113}$ The safety profile of tedizolid has been investigated in both the ESTABLISH studies and the most common adverse events were mild such as nausea, headache and abscess and they were reported in the tedizolid group less frequently than in the linezolid one (16\% vs 23\%). Severe side effects that required the discontinuation of the therapy occurred in less than $1 \%$ in both arms. Notably, a lower percentage of patients in the tedizolid group experienced a platelet count reduction $(6.4 \%$ in the tedizolid group vs $12.6 \%$ in the linezolid one), under $150,000 / \mathrm{mmc}$. Furthermore, recently the findings of a phase 1 study comparing increasing doses of tedizolid $(200,200,400 \mathrm{mg}$ every $24 \mathrm{~h}$, respectively), standard dose of linezolid (600 mg every $2 \mathrm{~h}$ ) and placebo in healthy subjects, supported further clinical studies of extended treatment duration with tedizolid since for tedizolid, mean platelets counts decreased over time in a dose-dependent manner and abnormal hematological values were observed only in those participants assuming scheduled dosage of linezolid or the highest dosage $(400 \mathrm{mg} / \mathrm{die})$ of tedizolid. ${ }^{114}$ Moreover, another phase-1 study, with the aim to assess the effects of tedizolid on the QTc interval, compared tedizolid, using both a single therapeutic dose $(200 \mathrm{mg})$ and a supratherapeutic one (1200 mg), to moxifloxacin in healthy adults. The results of this study demonstrated that even the higher dosage of tedizolid had fewer adverse events than moxifloxacin and that those occurred were generally mild and clinically insignificant. ${ }^{115}$ In addition, differently from linezolid, tedizolid does not appear to inhibit, in a substantial way, the monoamine oxidase system and thus it could be considered as an alternative treatment in patients with concomitant use of serotoninergic agents. ${ }^{116}$

Furthermore, since many studies have already assessed the high pulmonary diffusion of tedizolid, ${ }_{117,118}$ currently, it is ongoing a phase-3, double-blind, randomized clinical trial with the aim to assess the clinical efficacy of a 7-day course of tedizolid (200 mg iv every $24 \mathrm{~h}$ ) compared to a 10 -day 
Table 5. US Food and Drug Administration and European Medicines Agency indications and off-label possible indications for each novel antibiotic described above.

\begin{tabular}{|c|c|c|}
\hline Antibiotic & FDA approved-indication & Possible off-label indication \\
\hline Ceftolozane/tazobactam & cUTI, cIAI & $\begin{array}{l}\text { HAP/VAP, severe and deep infections } \\
\text { due to suspected MDR Gram-negative bacteria }\end{array}$ \\
\hline Ceftazidime/avibactam & $\begin{array}{c}\text { cUTI, cIAI (EMA: also for HAP/VAP } \\
\text { and severe Gram-negative infections } \\
\text { with no other therapeutic options available) }\end{array}$ & $\begin{array}{l}\text { HAP/VAP, severe and deep infections } \\
\text { due to MDR Gram-negative bacteria }\end{array}$ \\
\hline Eravacycline & Approval is still pending & $\begin{array}{l}\text { cUTI, cIAI, HAP/VAP, severe and deep infections } \\
\text { due to suspected MDR Gram-negative bacteria }\end{array}$ \\
\hline Plazomicin & Approval is still pending & $\begin{array}{l}\text { cUTI, sepsis and HAP/VAP } \\
\text { due to carbapenem-resistant Gram-negative bacteria }\end{array}$ \\
\hline Dalbavancin & ABSSSI & $\begin{array}{l}\text { Osteomyelitis, sepsis and endocarditis } \\
\text { due to Gram-positive pathogens }\end{array}$ \\
\hline Oritavancin & ABSSSI & $\begin{array}{l}\text { Osteomyelitis, sepsis and endocarditis } \\
\text { due to Gram-positive pathogens }\end{array}$ \\
\hline Tedizolid & ABSSSI & Osteomyelitis, HAP/VAP, CNS infections \\
\hline
\end{tabular}

FDA, Food and Drug Administration; cUTI, complicated urinary tract infections; cIAI, complicated intra-abdominal infections; HAP, hospital acquired pneumonia; VAP, ventilator-associated pneumonia; EMA, European Medicines Agency; MDR, multi-drug resistant bacteria; ABSSSI, acute bacterial skin and skin structure infections; CNS, central nervous system.

course of linezolid (600 mg iv every $12 \mathrm{~h}$ ) for the treatment of ventilated patients with presumed grampositive hospital-acquired pneumonia or ventilatorassociated pneumonia (in both arms, subjects with concurrent gram-positive bacteremia must receive a 14-day course of active treatment). ${ }^{119}$ To date this study is still recruiting participants and primary data are not expected before late 2018 .

Although, nowadays, tedizolid could be used only for the treatment of adult cutaneous infections (ABSSSI), ${ }^{120,121}$ its pharmacological characteristics encourage clinicians to consider its use for the treatment of other infections due to Gram-positive bacteria or for patients who cannot bear a multiple-daily dosing regimen or in whom an oral regimen is preferred.

\section{Discussion and Conclusions}

Advances in the treatment and prevention of infectious diseases are one of the greatest gains in medicine of the past century and this, at least in part, is due to antibiotic discovery and development. ${ }^{122}$ In recent years, the spread of multidrug-resistant pathogens and the presence of a weak antibiotic pipeline have led to calls for a variety of incentives to spur development of new antibiotic, particularly those against multidrugresistant bacteria.

In the last few years, the FDA and the EMA approved many new antibiotic molecules while for others approval is still pending. As presented in this article, the majority of the recently approved antibiotics belong to an already-existing drug classes, first of all $\alpha$-lactams and glycopeptides, thus lacking in biological innovation or public health importance. ${ }^{123}$ In addition, most of registrational trials of these new antibiotics were designed as non-inferiority trial, so that it is not possible to assess the superiority of these newer regimens than those already existing, Indeed, none of these trials evaluated direct patient outcomes as primary endpoint (patient mortality and patient disability) and the assessment of clinical cure and clinical response were often based on clinician's personal judgment.

Moreover, many of the novel antibiotics have the same indications, as showed in Table 5.

Concluding, as antibiotic research and development need to move forward, greater attention needs to be focused on new molecules, with new mechanism of actions, highly active on multidrug-resistant organism, available in oral formulations or in single-shot regimens and with demonstrated superiority to existing antibiotics in clinical trials.

Only in this way the gains in medicine of next decades would be as great as the one we had in the $20^{\text {th }}$ century.

\section{References}

1. Centers for Disease Prevention and Control (CDC). Antibiotic resistance threats in the United States; 2013. Available from: http:/www.cdc.gov/drugresistance/ threat-report-2013/index.html

2. Gross AE, Van Schooneveld TC, Olsen KM, et al. Epidemiology and predictors of multidrug-resistant community-acquired and healthcare-associated pneumonia. Antimicrob Agents Chemother 2014;58:5262-8.

3. Kwa AL, Low JG, Lee E, et al. The impact of multidrug resistance on the outcomes of critically ill patients with 
Gram-negative bacterial pneumonia. Diagn Microbiol Infect Dis 2007;58:99-104.

4. Toda A, Ohki H, Yamanaka T, et al. Synthesis and SAR of novel parenteral anti-pseudomonal cephalosporins: discovery of FR264205. Bioorg Med Chem Lett 2008; 18:4849-52.

5. Takeda S, Nakai T, Wakai Y, et al. In vitro and in vivo activities of a new cephalosporin, FR264205, against Pseudomonas aeruginosa. Antimicrob Agents Chemother 2007;51:826-30.

6. Zhanel GG, Chung P, Adam H, et al. Ceftolozane/ tazobactam: a novel cephalosporin/ $\beta$-lactamase inhibitor combination with activity against multidrug-resistant gram-negative bacilli. Drugs 2014;74:31-51.

7. Toussaint KA, Gallagher JC. $\beta$-lactam/ $\beta$-lactamase inhibitor combinations: from then to now. Ann Pharmacother 2015;49:86-98.

8. Lepak AJ, Reda A, Marchillo K, et al. Impact of MIC range for Pseudomonas aeruginosa and Streptococcus pneumoniae on the ceftolozane in vivo pharmacokinetic/ pharmacodynamic target. Antimicrob Agents Chemother 2014;58:6311-4.

9. Ge Y, Whitehouse MJ, Friedland I, et al. Pharmacokinetics and safety of CXA-101, a new antipseudomonal cephalosporin, in healthy adult male and female subjects receiving single- and multiple-dose intravenous infusions. Antimicrob Agents Chemother 2010;54:3427-31.

10. Miller B, Hershberger E, Bezinger D, et al. Pharmacokinetics and safety of intravenous ceftolozane-tazobactam in healthy adult subjects following single and multiple ascending doses. Antimicrob Agents Chemother 2012;56: 3086-91.

11. Chandorkar G, Xiao A, Mouksassi M-S, et al. Population pharmacokinetics of ceftolozane/tazobactam in healthy volunteers, subjects with varying degrees of renal function and patients with bacterial infections. J Clin Pharmacol 2015;55:230-9.

12. Wooley M, Miller B, Krishna G, et al. Impact of renal function on the pharmacokinetics and safety of ceftolozane-tazobactam. Antimicrob Agents Chemother 2014;58:2249-55.

13. Farrell DJ, Flamm RK, Sader HS, et al. Antimicrobial activity of ceftolozane-tazobactam tested against Enterobacteriaceae and Pseudomonas aeruginosa with various resistance patterns isolated in U.S. Hospitals (2011-2012). Antimicrob Agents Chemother 2013;57: 6305-10.

14. Solomkin J, Hershberger E, Miller B, et al. Ceftolozane/tazobactam plus metronidazole for complicated intra-abdominal infections in an era of multidrug resistance: results from a randomized, double-blind, phase 3 trial (ASPECT-cIAI). Clin Infect Dis 2015;60: 1462-71.

15. Wagenlehner FM, Umeh O, Steenbergen $\mathrm{J}$, et al. Ceftolozane-tazobactam compared with levofloxacin in the treatment of complicated urinary-tract infections, including pyelonephritis: a randomised, double-blind, phase 3 trial (ASPECT-cUTI). Lancet 2015;385:1949-56.

16. ClinicalTrials.gov. Safety and efficacy study of ceftolozane/tazobactam to treat ventilated nosocomial pneumonia (MK-7625A-008) (ASPECT-NP). Available from: https://clinicaltrials.gov/ct2/show/NCT 02070757
17. Cubist Pharmaceuticals U.S.. ZERBAXA (ceftolozane/ tazobactam) for Injection, for intravenous use Initial U.S. Approval: 2014. Available from: http://www.accessdata. fda.gov/drugsatfda_docs/label/2014/206829lbl.pdf

18. Patel UC, Nicolau DP, Sabzwari RK. Successful treatment of multi-drug resistant pseudomonas aeruginosa bacteremia with the recommended renally adjusted ceftolozane/tazobactam regimen. Infect Dis Ther 2016; 5:73-9.

19. Aitken SL, Kontoyiannis DP, DePombo AM, et al. Use of ceftolozane/tazobactam in the treatment of multidrug-resistant Pseudomonas aeruginosa bloodstream infection in a pediatric leukemia patient. Pediatr Infect Dis J 2016;35:1040-2.

20. Coleman K. Diazabicyclooctanes (DBOs): a potent new class of non- $\beta$-lactam $\beta$-lactamase inhibitors. Curr Opin Microbiol 2011;14:550-5.

21. Zhanel GG, Lawson CD, Adam H, et al. Ceftazidimeavibactam: a novel cephalosporin/ $\beta$-lactamase inhibitor combination. Drugs 2013;73:159-77.

22. Levasseur P, Girard AM, Miossec C, et al. In vitro antibacterial activity of the ceftazidime-avibactam combination against enterobacteriaceae, including strains with well-characterized $\beta$-lactamases. Antimicrob Agents Chemother 2015;59:1931-4.

23. Levasseur P, Girard AM, Claudon M, et al. In vitro antibacterial activity of the ceftazidime-avibactam (NXL104) combination against Pseudomonas aeruginosa clinical isolates. Antimicrob Agents Chemother 2012;56:1606-8.

24. Sader HS, Castanheira M, Flamm RM, et al. Ceftazidime-avibactam activity against aerobic gram negative organisms isolated from intra-abdominal infections in United States hospitals, 2012-2014. Antimicrob Agents Chemother 2014;53:1684-92.

25. Sader HS, Castanheira M, Mendes RE, et al. Ceftazidime-avibactam activity against multidrug-resistant Pseudomonas aeruginosa isolated in U.S. medical centers in 2012 and 2013. Antimicrob Agents Chemother 2015;59:3656-9.

26. Citron DM, Tyrrell KL, Merriam V, et al. In vitro activity of ceftazidime-NXL104 against 396 strains of $\alpha$ lactamase-producing anaerobes. Antimicrob Agents Chemother 2011;55:3616-20.

27. Merdjan H, Rangaraju M, Tarral A. Safety and pharmacokinetics of single and multiple ascending doses of avibactam alone and in combination with ceftazidime in healthy male volunteers: results of two randomized, placebo-controlled studies. Clin Drug Investig 2015; 35:307-17.

28. Tominaga N, Edeki T, Li J, et al. Phase I study assessing the safety, tolerability, and pharmacokinetics of avibactam and ceftazidime-avibactam in healthy Japanese volunteers. J Infect Chemother 2015;21:551-8.

29. Vazquez JA, González Patzán LD, Stricklin D, et al. Efficacy and safety of ceftazidime-avibactam versus imipenem-cilastatin in the treatment of complicated urinary tract infections, including acute pyelonephritis, in hospitalized adults: results of a prospective, investigator-blinded, randomized study. Curr Med Res Opin 2012;28:1921-31.

30. Mazuski JE, Gasink L, Armstrong J, et al. Efficacy and safety of ceftazidime-avibactam plus metronidazole 
versus meropenem in the treatment of complicated intra-abdominal infection: results from a randomized, controlled, double-blind, phase 3 program. Clin Infect Dis 2016;62:1380-9.

31. ClinicalTrials.Gov. Ceftazidime-avibactam compared with doripenem followed by oral therapy for hospitalized adults with complicated UTIs (urinary tract infections); 2012-2016. Available from: https:/clinicaltrials. gov/ct2/show/NCT01599806?term=ceftazidime+aviba ctam + cuti + phase $+3 \&$ rank $=1$

32. ClinicalTrials.Gov. Ceftazidime-avibactam compared with doripenem followed by oral therapy for hospitalized adults with complicated UTIs (urinary tract infections); 2012-2016. Available from: https://clinicaltrials. gov/ct2/show/NCT01595438?term $=$ ceftazidime+aviba ctam + cuti + phase $+3 \&$ rank $=2$

33. Lucasti C, Popescu I, Ramesh MK, et al. Comparative study of the efficacy and safety of ceftazidime/avibactam plus metronidazole versus meropenem in the treatment of complicated intra-abdominal infections in hospitalized adults: results of a randomized, double-blind, Phase II trial. J Antimicrob Chemother 2013;68:1183-92.

34. Carmeli Y, Armstrong J, Laud PJ, et al. Ceftazidimeavibactam or best available therapy in patients with ceftazidime-resistant Enterobacteriaceae and P. aeruginosa cUTIs or cIAIs (REPRISE): a randomised, pathogen-directed, phase 3 study. Lancet Infect Dis 2016;16:661-73.

35. ClinicalTrials.Gov. A study comparing ceftazidimeavibactam versus meropenem in hospitalized adults with nosocomial pneumonia; 2013-2016. Available from: https://clinicaltrials.gov/ct2/show/study/NCT01808092?t erm $=$ ceftazidime + avibactam + phase $+3 \&$ rank $=2$

36. ClinicalTrials.Gov. A study of avycaz (ceftazidime/ avibactam) pharmacokinetics/pharmacodynamics (PK/PD) in critically ill patients. Available from: https://clinicaltrials.gov/ct2/show/NCT02822950?term =ceftazidime+a vibactam + phase $+3 \&$ rank $=15$

37. ClinicalTrials.Gov. Steady-state pharmacokinetics of ceftazidime/avibactam in cystic fibrosis; 2015-2016. Available from: https://clinicaltrials.gov/ct2/show/ NCT02504827?term $=$ ceftazidime + avibactam + phase +3 \&rank $=13$

38. ClinicalTrials.Gov. Study to assess the concentration of avibactam and ceftazidime in epithelial lining fluid and plasma; 2011-2016. Available from: https://clinicaltrials.gov/ct2/show/NCT01395420?term=ceftazidime + av ibactam + phase +3 \& rank $=9$

39. ClinicalTrials.Gov. Evaluation of safety, pharmacokinetics and efficacy of CAZ-AVI with metronidazole in childern aged 3 months to 18 years old with complicated intra-abdominal infections (cIAIs). Available from: https://clinicaltrials.gov/ct2/show/NCT02475733?term= ceftazidime + avibactam + phase $+3 \&$ rank $=11$

40. ClinicalTrials.Gov. Evaluation of safety, pharmacokinetics and efficacy of ceftazidime and avibactam (CAZAVI) Compared with cefepime in children from 3 months to less than 18 years of age with complicated urinary tract infections (cUTIs). Available from: https:/clinicaltrials.gov/ct2/show/NCT02497781?term $=$ ceftazidime + avibactam + phase $+3 \&$ rank $=12$

41. European Medicines Agency (EMA). Ceftazidime/ avibactam (Zavicefta): summary of product characteristics. Available from: http://www.ema.europa.eu/docs/
en_GB/document_library/EPAR_-_Product_Information/human/004027/WC500210234.pdf

42. U.S Food and Drug Administration (FDA). Ceftazidime/avibactam (Avycaz): prescribing information. Available from: http://www.accessdata.fda.gov/ drugsatfda_docs/label/2016/206494s002lbl.pdf

43. Gallagher JC, Cprek J, Famon E, et al. Compassionate use ceftazidime/avibactam (CAZ-AVI) for carbapenem-resistant Klebsiella pneumoniae (CRKP) infections. Abstract presented at: 18th Making a Difference in Infectious Diseases (MAD-ID) Meeting, 2015, Orlando, FL, USA.

44. Camargo JF, Simkins J, Beduschi T, et al. Successful treatment of carbapenemase-producing pandrug-resistant Klebsiella pneumoniae bacteremia. Antimicrob Agents Chemother 2015;59:5903-8.

45. Xiao XY, Hunt DK, Zhou J, et al. Fluorocyclines. 1. 7fluoro-9-pyrrolidinoacetamido-6-demethyl-6-deoxytetracycline: a potent, broad spectrum antibacterial agent. J Med Chem 2012;55:597-605.

46. Clark RB, Hunt DK, He M, et al. Fluorocyclines. 2. Optmization of the C-9 side-chain for antibacterial activity and oral efficacy. J Med Chem 2012;55:606-22.

47. Sutcliffe JA, O'Brien W, Fyfe C, et al. Antibacterial activity of eravacycline (TP-434), a novel fluorocycline, against hospital and community pathogens. Antimicrob Agents Chemother 2013;57:5548-58.

48. Abdallah M, Olafisoye O, Cortes C, et al. Activity of eravacycline against Enterobacteriaceae and Acinetobacter baumannii, including multidrug-resistant isolates, from New York City. Antimicrob Agents Chemother 2015;59:1802-5.

49. Livermore DM, Mushtaq S, Warner M, et al. In vitro activity of eravacycline against carbapenem-resistant Enterobacteriaceae and Acinetobacter baumannii. Antimicrob Agents Chemother 2016;60:3840-4.

50. Zhang Y, Lin X, Bush K. In vitro susceptibility of $\beta$ lactamase-producing carbapenem-resistant Enterobacteriaceae (CRE) to eravacycline. J Antibiot (Tokyo) 2016;69:600-4.

51. Thabit AK, Monogue ML, Nicolau DP. Eravacycline pharmacokinetics and challenges in defining humanized exposure in vivo. Antimicrob Agents Chemother 2016;60:5072-5.

52. Monogue ML, Thabit AK, Hamada Y, et al. Antibacterial efficacy of eravacycline in vivo against gram-positive and gram-negative organisms. Antimicrob Agents Chemother 2016;60:5001-5.

53. Leighton A, Zupanets I, Bezugla N, et al. Broad spectrum fluorocycline TP-434 has oral bioavailability in humans (abstract $\mathrm{n}^{\circ} \mathrm{P} 1509$ plus poster). 21 st European Congress of Clinical Microbiology and Infectious Diseases/27th International Congress of Chemotherapy 710 May 2011, Milan, Italy.

54. Connors KP, Housman ST, Pope JS, et al. Phase I, open-label, safety and pharmacokinetic study to assess bronchopulmonary disposition of intravenous eravacycline in healthy men and women. Antimicrob Agents Chemother 2014;58:2113-8.

55. Solomkin JS, Ramesh MK, Cesnauskas G, et al. Phase 2, randomized, double-blind study of the efficacy and safety of two dose regimens of eravacycline versus ertapenem for adult community-acquired complicated 
intra-abdominal infections. Antimicrob Agents Chemother 2014;58:1847-54.

56. ClinicalTrials.Gov. Efficacy and safety study of eravacycline compared with ertapenem in complicated intraabdominal infections (IGNITE1); 2013-2016. Available from: https://clinicaltrials.gov/ct2/show/results/NCT0 1844856 ? sect $=$ X70156\&term $=$ eravacycline $\&$ rank $=1 \#$ o utcome 1

57. ClinicalTrials.Gov. Efficacy and safety study of eravacycline compared with levofloxacin in complicated urinary tract infections; 2013-2016. Available from: https://clinicaltrials.gov/ct2/show/NCT01978938?term $=$ eravacycline \& $\mathrm{rank}=6$

58. Tsai L, March A, Lloyd K, et al. Results of the lead-in study on the safety and efficacy of eravacycline versuss levofloxacin in complicated urinary tract infections/abstract $^{\circ}$ 0198). 25th European Congress of Clinical Microbiology and Infectious Diseases, 25-28 Apr 2015, Copenhagen, Denmark.

59. Grossman TH, Murphy TM, Slee AM, et al. Eravacycline (TP-434) is efficacious in animal models of infection. Antimicrob Agents Chemother 2015;59:2567-71.

60. Galani I, Souli M, Daikos GL, et al. Activity of plazomicin (ACHN-490) against MDR clinical isolates of Klebsiella pneumoniae, Escherichia coli, and Enterobacter spp. from Athens, Greece. J Chemother 2012; 24:191-4.

61. Almaghrabi R, Clancy CJ, Doi J, et al. Carbapenem-resistant Klebsiella pneumoniae strains exhibit diversity in aminoglycoside-modifying enzymes, which exert differing effects on plazomicin and other agents. Antimicrob Agents Chemother 2014;58:4443-51.

62. Zhanel GG, Lawson CD, Zelenitsky S, et al. Comparison of the next-generation aminoglycoside plazomicin to gentamicin, tobramycin and amikacin. Expert Rev Anti Infect Ther 2012;10:459-73.

63. Garcia-Salguero C, Rodriguez-Avial I, Picazo JJ, et al. Can plazomicin alone or in combination be a therapeutic option against carbapenem-resistant Acinetobacter baumannii? Antimicrob Agents Chemother 2015;59: 5959-66.

64. Cass RT, Brooks CD, Havrilla NA, et al. Pharmacokinetics and safety of single and multiple doses of ACHN-490 injection administered intravenously in healthy subjects. Antimicrob Agents Chemother 2011; 55:5874-80.

65. Poulikakos P, Falagas ME. Aminoglycoside therapy in infectious diseases. Expert Opin Pharmacother 2013; 14:1585-97.

66. ClinicalTrials.Gov. A study of plazomicin compared with colistin in patients with infection due to carbapenem-resistant Enterobacteriaceae (CRE) (CARE); 2013-2016. Available from: https://clinicaltrials.gov/ ct2/show/NCT01970371?term=plazomicin\&rank=1

67. Malabarba A, Goldtein BP. Origin, structure, and activity in vitro and in vivo of dalbavancin. J Antimicrob Chemother 2005;55:ii15-20.

68. Chen AY, Zervos MJ, Vazquez JA. Dalbavancin: a novel antimicrobial. Int J Clin Pract 2007;61:853-63.

69. Buckwalter M, Dowell JA. Population pharmacokinetic analysis of dalbavancin, a novel lipoglycopeptide. J Clin Pharmacol 2005;45:1279-87.

70. Dorr MB, Jabes D, Cavaleri M, et al. Human pharma- cokinetics and rationale for once-weekly dosing of dalbavancin, a semi-synthetic glycopeptide. J Antimicrob Chemother 2005;55:ii25-30.

71. Nicolau D, Sun H, Seltzer E, et al. Pharmacokinetics of dalbavancin in plasma and skin blister fluid. J Antimicrob Chemother 2007;60:681-4.

72. Streit JM, Fritsche TR, Sader HS, et al. Worldwide assessment of dalbavancin activity and spectrum against over 6,000 clinical isolates. Diagn Microbiol Infect Dis 2004;48:137-43.

73. Goldstein EJ, Citron DM, Merriam CV, et al. In vitro activities of dalbavancin and nine comparator agents against anaerobic gram-positive species and corynebacteria. Antimicrob Agents Chemother 2003;47:1968-71.

74. Johnson DM, Fritsche TR, Sader HS, et al. Evaluation of dalbavancin in combination with nine antimicrobial agents to detect enhanced or antagonistic interactions. Int J Antimicrob Agents 2006;27:557-60.

75. Jauregui LE, Babazadeh S, Seltzer E, et al. Randomized, double-blind comparison of once-weekly dalbavancin versus twice-daily linezolid therapy for the treatment of complicated skin and skin structure infections. Clin Infect Dis 2005;41:1407-15.

76. Boucher HW, Wilcox M, Talbot GH, et al. Onceweekly dalbavancin versus daily conventional therapy for skin infection. N Engl J Med 2014;370:2169-79.

77. U.S. Food and Drugs Administration (FDA). DALVANCE (dalbavancin) for injection, for intravenous use Initial U.S. Approval: 2014. Available from: http:// www.accessdata.fda.gov/drugsatfda_docs/label/2016/0 21883s003lbl.pdf

78. European Medicines Agency (EMA). Dalbavancin (Xydalba): summary of product characteristics. Available from: http://www.ema.europa.eu/docs/en_GB/document_library/EPAR___Product_Information/human/0 02840/WC500183869.pdf

79. ClinicalTrials.Gov. Study of dalbavancin drug levels achieved in hospitalized adolescents who are receiving antibiotic therapy for bacterial infections; 2008-2009. Available from: https://clinicaltrials.gov/ct2/show/ NCT00678106? term $=$ dalbavancin\&rank $=10$

80. ClinicalTrials.Gov. A single dose study to investigate the pharmacokinetics and safety of dalbavancin in hospitalized children aged 3 months to 11 years; 20132015. Available from: https://clinicaltrials.gov/ct2/ show/NCT01946568?term=dalbavancin\&rank=3

81. ClinicalTrials. Gov. Study evaluate the pk profile of dalbavancin in hospitalized infants and neonates patients with known or suspected bacterial infection. Available from: https:/clinicaltrials.gov/ct2/show/NCT02688790 ?term=dalbavancin\&rank=2

82. Zhanel GG, Schweizer F, Karlowsky JA. Oritavancin: mechanism of action. Clin Infect Dis 2012;54:S214-9.

83. Belley A, Lalonde Seguin D, Arhin F, et al. Comparative in vitro activities of oritavancin, dalbavancin, and vancomycin against methicillin-resistant Staphylococcus aureus isolates in a nondividing state. Antimicrob Agents Chemother 2016;60:4342-5.

84. Mendes RE, Farrell DJ, Sader HS, et al. Activity of oritavancin against Gram-positive clinical isolates responsible for documented skin and soft-tissue infections in European and US hospitals (2010-13). J Antimicrob Chemother 2015;70:498-504. 
85. Mendes RE, Castanheira M, Farrell DJ, et al. Longitudinal (2001-14) analysis of enterococci and VRE causing invasive infections in European and US hospitals, including a contemporary (2010-13) analysis of oritavancin in vitro potency. J Antimicrob Chemother 2016 [Epub ahead of print].

86. Mendes RE, Sader HS, Flamm RK, et al. Oritavancin activity against Staphylococcus aureus causing invasive infections in U.S. and European hospitals: a 5-year international surveillance program. Antimicrob Agents Chemother 2014;58:2921-4.

87. Rubino CM, Van Wart SA, Bhavnani SM, et al. Oritavancin population pharmacokinetics in healthy subjects and patients with complicated skin and skin structure infections or bacteremia. Antimicrob Agents Chemother 2009;53:4422-8.

88. Bhavnani SM, Owen JS, Loutit JS, et al. Pharmacokinetics, safety, and tolerability of ascending single intravenous doses of oritavancin administered to healthy human subjects. Diagn Microbiol Infect Dis 2004;50: 95-102.

89. The Medicines Company. ORBACTIV® (oritavancin) prescribing information. Parsippany, NJ: The Medicines Company; 2016.

90. McKay GA, Beaulieu S, Arhin FF, et al. Time-kill kinetics of oritavancin and comparator agents against Staphylococcus aureus, Enterococcus faecalis and Enterococcus faecium. J Antimicrob Chemother 2009;63: 1191-9.

91. Corey GR, Kabler H, Mehra P, et al. Single-dose oritavancin in the treatment of acute bacterial skin infections. N Engl J Med 2014;370:2180-90.

92. Corey GR, Good S, Jiang H, et al. Single-dose oritavancin versus 7-10 days of vancomycin in the treatment of gram-positive acute bacterial skin and skin structure infections: the SOLO II noninferiority study. Clin Infect Dis 2015;60:254-62.

93. Brade KD, Rybak JM, Rybak, MJ. Oritavancin: a new lipoglycopeptide antibiotic in the treatment of grampositive infections. Infect Dis Ther 2016;5:1-15.

94. ClinicalTrials.Gov. A study to evaluate the safety of a single iv dose of Orbactiv (oritavancin) in subjects on chronic warfarin therapy being treated for acute bacterial skin and skin structure infection (ABSSSI); 20152016. Available from: https:/clinicaltrials.gov/ct2/ show/NCT02452918?term $=$ oritavancin\&rank $=4$

95. ClinicalTrials.Gov. Open label study evaluating the effects of a single oritavancin infusion on cytochrome P450 in healthy volunteers (DDI); 2013. Available from: https://clinicaltrials.gov/ct2/show/NCT01784536 ?term $=$ oritavancin\&rank $=2$

96. U.S. Food and Drug Administration (FDA). ${ }^{\circledR}$ ORBACTIV (oritavancin) for injection, for intravenous use Initial U.S. Approval: 2014. Available from: http://www.accessdata.fda.gov/drugsatfda_docs/label/2 016/206334s0021bl.pdf

97. European Medicines Agency (EMA). Oritavancin (Orbactiv): summary of product characteristics. Available from: http://www.ema.europa.eu/docs/en_GB/document_library/EPAR___Product_Information/human/ 003785/WC500186343.pdf

98. McCusker KP, Fujimori DG. The chemistry of peptidyltransferase center-targeted antibiotics: enzymatic resistance and approaches to countering resistance. ACS Chem Biol 2012;7:64-72.

99. Im WB, Choi SH, Park JY, et al. Discovery of torezolid as a novel 5-hydroxymethyl-oxazolidinone antibacterial agent. Eur J Med Chem 2011;46:1027-39.

100. Locke JB, Finn J, Hilgers M, et al. Structure-activity relationships of diverse oxazolidinones for linezolidresistant Staphylococcus aureus strains possessing the cfr methyltransferase gene or ribosomal mutations. Antimicrob Agents Chemother 2010;54:5337-43.

101. Shaw KJ, Poppe S, Schaadt R, et al. In vitro activity of TR-700, the antibacterial moiety of the prodrug TR701, against linezolid-resistant strains. Antimicrob Agents Chemother 2008;52:4442-7.

102. Jones RN, Moet GJ, Sader HS, et al. TR-700 in vitro activity against and resistance mutation frequencies among Gram-positive pathogens. J Antimicrob Chemother 2009;63:716-20.

103. Barber KE, Smith JR, Raut A, et al. Evaluation of tedizolid against Staphylococcus aureus and enterococci with reduced susceptibility to vancomycin, daptomycin or linezolid. J Antimicrob Chemother 2016;71:152-5.

104. LaMarre JM, Locke JB, Shaw KJ, et al. Low fitness cost of the multidrug resistance gene cfr. Antimicrob Agents Chemother 2011;55:3714-9.

105. Smith LK, Mankin AS. Transcriptional and translational control of the mlr operon, which confers resistance to seven classes of protein synthesis inhibitors. Antimicrob Agents Chemother 2008;52:1703-12.

106. Locke JB, Rahawi S, LaMarre J, et al. Genetic environment and stability of cfr in methicillin-resistant Staphylococcus aureus CM05. Antimicrob Agents Chemother 2012;56:332-40.

107. Li S, Guo Y, Zhao C, et al. In vitro activities of tedizolid compared with other antibiotics against gram-positive pathogens associated with hospital acquired pneumonia (HAP), skin and soft tissue infection (SSTI) and bloodstream infection (BSI) collected from hospitals in China. J Med Microbiol 2016 [Epub ahead of print].

108. Klupp EM, Both A, Belmar Campos C, et al. Tedizolid susceptibility in linezolid- and vancomycin-resistant Enterococcus faecium isolates. Eur J Clin Microbiol Infect Dis 2016 [Epub ahead of print].

109. Jones RN, Holliday NM, Rhomberg PR. Validation of a commercial dry-form broth microdilution device (Sensititre) for testing tedizolid, a new oxazolidinone. J Clin Microbiol 2015;53:657-9.

110. Merck \& Co., Inc. Tedizolid (Sivextro); 2016. Available from: http://sivextro.com

111. Pai MP. Pharmacokinetics of tedizolid in morbidly obese and covariate-matched nonobese adults. Antimicrob Agents Chemother 2016;60:4585-9.

112. Prokocimer P, De Anda C, Fang E, et al. Tedizolid phosphate vs linezolid for treatment of acute bacterial skin and skin structure infections: the ESTABLISH-1 randomized trial. JAMA 2013;309:559-69.

113. Moran GJ, Fang E, Corey GR, et al. Tedizolid for 6 days versus linezolid for 10 days for acute bacterial skin and skin-structure infections (ESTABLISH-2): a randomised, double-blind, phase 3, non-inferiority trial. Lancet Infect Dis 2014;14:696-705.

114. Lodise TP, Bidell MR, Flanagan SD, et al. Characterization of the haematological profile of 21 days of tedi- 
zolid in healthy subjects. J Antimicrob Chemother 2016;71:2553-8.

115. Flanagan S, Litwin J, Fang E, et al. Effects of therapeutic and supratherapeutic doses of oral tedizolid phosphate on cardiac repolarisation in healthy volunteers: a randomised controlled study. Int J Antimicrob Agents 2016;48:33-40.

116. Flanagan S, Bartizal K, Minassian SL, et al. In vitro, in vivo, and clinical studies of tedizolid to assess the potential for peripheral or central monoamine oxidase interactions. Antimicrob Agents Chemother 2013;57:3060-6.

117. Housman ST, Pope JS, Russomanno J, et al. Pulmonary disposition of tedizolid following administration of once-daily oral 200-milligram tedizolid phosphate in healthy adult volunteers. Antimicrob Agents Chemother 2012;56:2627-34.

118. Lodise TP, Drusano GL. Use of pharmacokinetic/pharmacodynamic systems analyses to inform dose selection of tedizolid phosphate. Clin Infect Dis 2014;58:S28-34.
119. ClinicalTrials.Gov. TR-701 FA vs linezolid for the treatment of nosocomial pneumonia. Available from: https://clinicaltrials.gov/ct2/show/NCT02019420?term $=$ tedizolid\&rank $=21$

120. U.S. Food and Drug Administration (FDA). Tedizolid (Sivextro): prescriving information; 2016. Available from: http://www.accessdata.fda.gov/drugsatfda_docs/ label/2015/0205435s001,0205436s0011bl.pdf

121. European Medicines Agency (EMA). Tedizolid (Sivextro): summary of product characteristics; 2016. Available from: http://www.ema.europa.eu/docs/en_GB/ document_library/EPAR_-_Product_Information/ human/002846/WC500184802.pdf

122. Deak D, Outterson K, Powers JH, et al. Progress in the fight against multidrug-resistant bacteria? a review of U.S. Food and Drug Administration-approved antibiotics, 2010-2015. Ann Intern Med 2016;165:363-72.

123. Doshi P. Speeding new antibiotics to market: a fake fix?. BMJ 2015;350:h1453. 